\section{Preimplantation HLA typing for stem cell transplantation treat- ment of hemoglobinopathies}

\author{
Anver Kuliev, Oleg Verlinsky, Svetlana \\ Rechitsky \\ Reproductive Genetics Institute, Chicago \\ IL, USA
}

\section{Abstract}

Preimplantation genetic diagnosis (PGD) for HLA typing is steadily becoming an option for at risk couples with thalassemic children, requiring HLA matched bone marrow transplantation treatment. The paper presents the world's largest PGD experience of 475 cases for over 2 dozens thalassemia mutations, resulting in birth of 132 unaffected children. A total of 146 cases were performed together with preimplantation HLA typing, resulting in detection and transfer of HLA matched unaffected embryos in 83 of them, yielding the birth of 16 HLA matched children, potential donors for their affected siblings. The presented experience of HLA matched stem cell transplantation for thalassemia, following PGD demonstrated a successful hematopoietic reconstitution both for younger and older patients. The data show that PGD is an efficient approach for HLA matched stem cell transplantation treatment for thalassemia.

\section{Introduction}

Thalassemia continues to be one of the major indications for preimplantation genetic diagnosis (PGD) ${ }^{1,2}$ Although it was originally offered for those at risk who could not accept the risk for prenatal diagnosis and termination of pregnancy, ${ }^{3-5}$ increasing number of at risk couples request also preimplantation HLA typing to allow performing HLA compatible stem cell transplantation for existing thalassemic siblings..$^{6-9}$ We have previously reported the results of PGD for thalassemia, with simultaneous HLA typing, resulting in birth of thalassemia-free HLA matched children, as donors of cord blood and/or bone marrow for transplantation treatment for their affected siblings. ${ }^{10,11}$ The present paper describes the progress in PGD for hemoglobinopathies with preimplantation HLA typing, demonstrating that PGD has become an acceptable approach for HLA matched stem cell transplantation treatment for hemoglobinopathies.

\section{Materials and Methods}

A total of 475 PGD cycles were performed for 276 couples at risk for producing offspring with thalassemia and sickle cell disease (Table 1). PGD cycles were performed using a standard IVF protocol coupled with micromanipulation procedures for the polar body (PB) sampling, or embryo biopsy at cleavage or blastocyst stage, described in detail elsewhere. ${ }^{2,10,11}$ In brief, the oocytes were tested by sampling of the first and second polar bodies (PB1 and PB2), removed following maturation and fertilization of oocytes, using mechanical micromanipulation technique. The biopsied oocytes were then returned to culture, checked for cleavage and transferred, depending on the genotype of the corresponding PB1 and PB2, which, however, provide the diagnosis only for maternal mutations.

HLA typing or testing for paternal mutations was performed on embryos biopsy samples, obtained at the cleavage or blastocyst stage, in order to identify the embryos containing the maternal and paternal chromosomes 6 , identical to the sibling with thalassemia, as also described in detail elsewhere., ${ }^{2,6,11}$ In brief, HLA haplotypes were tested simultaneously with mutations, using the short tandem repeats (STR) in the HLA region, by applying a multiplex hemi-nested polymerase chain reaction system, involving only closely linked STRs throughout HLA region (D6S426, D6S291, Ring 3 CA, TAP1, G51152, D6S2447, LH1,DN, D6S273, 9N-2, TNF a,b,c,d; 62, MIC A, MIB, D6S276, D6S439, D6S1624, D6S265, D6S510; D6S248, RF, M0G a,b,c,d, D6S 258, D6S306, D6S464, D6S299, D6S461). The choice of linked markers was based on the information they provide about the presence of maternal and paternal matching or non-matching chromosomes. For each family heterozygous alleles were selected to be not shared by the parents. Such markers provided the information about the origin of chromosome 6 . The applied method provided the data for obtaining a 100\% HLA match, because the embryos with the same paternal and maternal chromosome 6 , as in the affected siblings, were preselected.

\section{Results and Discussion}

Our experience of PGD for hemoglobinopathies is presented in Table 1, representing the largest world's experience available in one center. At the present time, PGD for hemoglobinopathies is more than $15 \%$ of our overall experience of 2982 PGD cycles performed for single gene disorders, and involves testing of over two dozens of different HBB gene mutations. $^{2}$ Of 475 clinical cycles performed, unaf-
Correspondence: Anver Kuliev, Reproductive Genetics Institute, 2825 North Halsted Street, Chicago IL, 60657 USA.

Tel: 773.472.4900 - Fax: 773.871.5221.

E-mail: anverkuliev@hotmail.com

Key words: preimplantation genetic diagnosis for hemoglobinopathies, preimplantation HLA typing, stem cell transplantation.

Received for publication: 30 July 2013

Revision received: 23 December 2013.

Accepted for publication: 11 February 2014.

This work is licensed under a Creative Commons Attribution 3.0 License (by-nc 3.0).

(C) Copyright A. Kuliev et al., 2014

Licensee PAGEPress, Italy

Thalassemia Reports 2014; 4:1853

doi:10.4081/thal.2014.1853

fected embryos for transfer were detected in 372 (78.3\%) of them, resulting in 133 (30.8\%) clinical pregnancies and birth of 132 hemoglobinopathy-free children. As many as one quarter of PGD cycles were performed for IVS I-110 mutation, which is the most common thalassemia mutation in the Eastern Mediterranean region, and approximately one fifth for sickle cell disease, prevalent in African Americans in the USA.

PGD with HLA typing for thalassemia and sickle cell disease was performed in 146 cases (137 for thalassemia and 9 for sickle cell disease), which resulted in transfer of 130 unaffected HLA matched embryos (121 and 9 respectively) in 83 cycles, yielding 21 clinical pregnancies and birth of 16 unaffected HLA match children. The chances to identify unaffected embryos fully matched to thalassemic siblings is $18.75 \%$, based on $25 \%$ chance of HLA match and $75 \%$ chance of having unaffected embryo.

Figure 1 presents PGD for HLA with the birth of thalassemia free baby boy, who became a donor of HLA matched stem cells for his thalassemic sibling. Bone marrow transplantation was performed in the International Center for Transplantation in Thalassemia and Sickle Cell Anemia of the Mediterranean Institute of Hematology in Rome, Italy (Javid Gaziev, personal communication, 2008). At the time of transplantation, the patient was 14 years old, with regular blood transfusion and chelation therapy since he was diagnosed with double heterozygous (Cod39/IVSI-110) thalassemia major. Despite being at the class-3 risk, ${ }^{12}$ transplantation with bone marrow obtained from the HLA matched sibling shown in Figure 1 resulted in a complete hematopoietic reconstitution.

The above experience is only a part of our overall world's largest experience of 474 cases of preimplantation HLA typing, which resulted in detection and transfer of HLA matched unaf- 
A

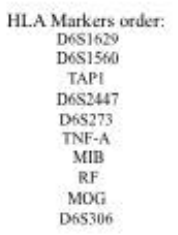

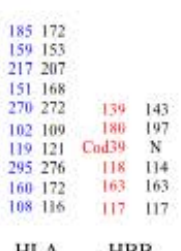

HLA HBB

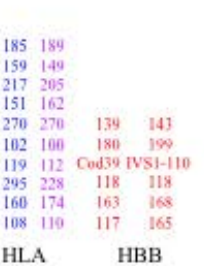

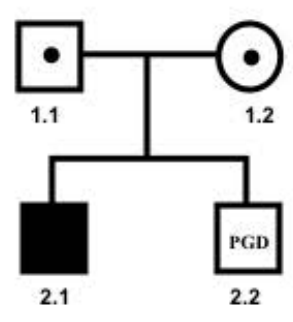

$\frac{\text { GD }}{2.2}$
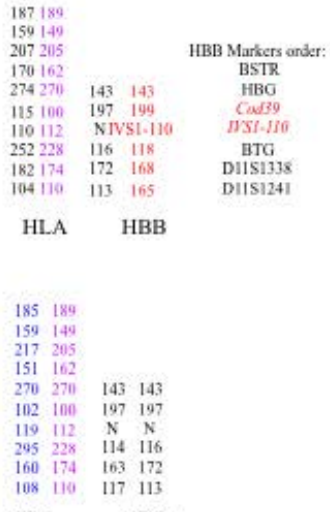

B

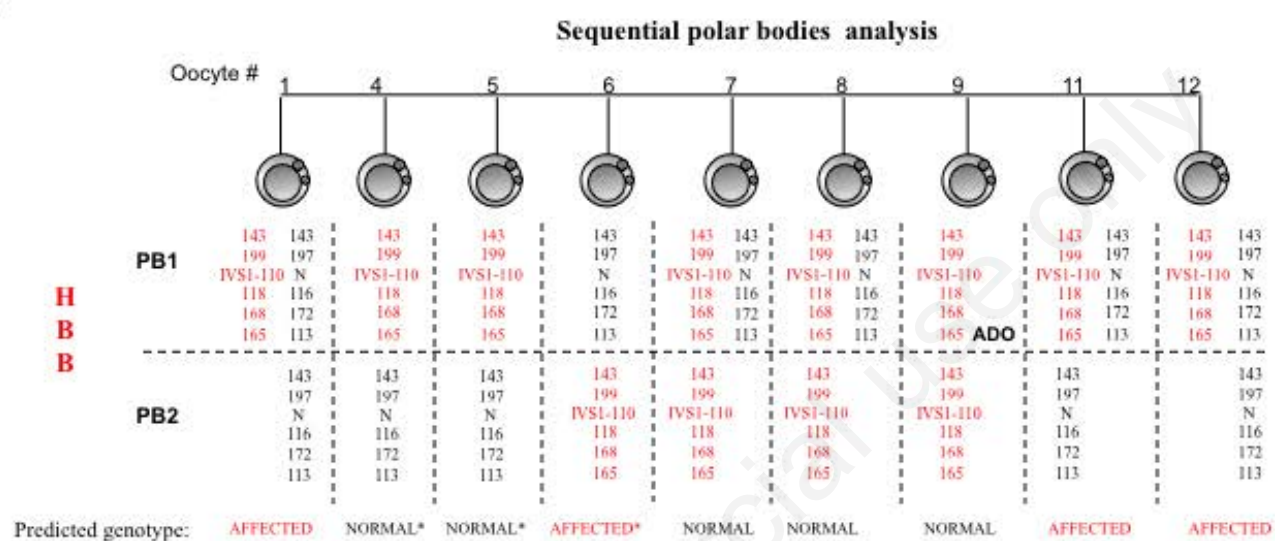

C

Blstomeres analysis
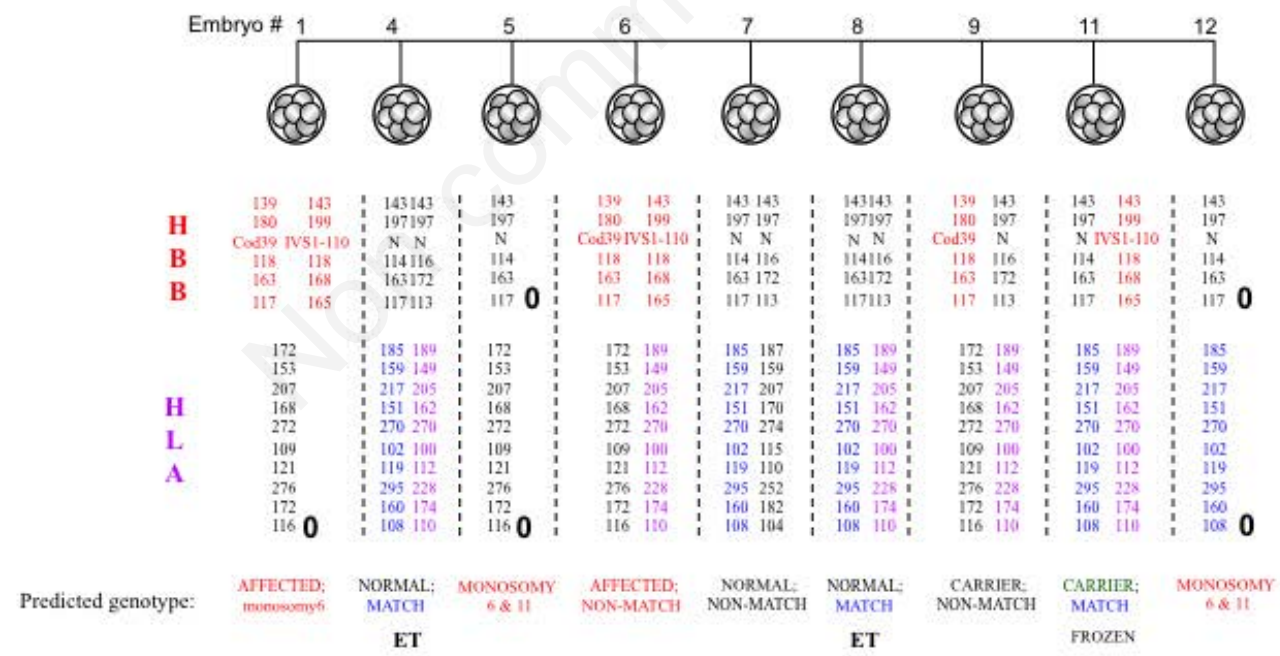

Figure 1. Preimplantation genetic diagnosis (PGD) for HLA typing in couple with thalassemic child, requiring HLA matched bone marrow transplantation. A) Family pedigree with HLA haplotype analysis based on parental (1.1 and 1.2) and affected child (2.1) genomic DNA testing. HLA markers order is presented on the upper left for father and right for mother. Paternal and maternal matching HLA haplotypes to the affected child (2.1) are shown in different colors. Maternal (IVS1-110) and paternal (Cod39) mutations and the linked markers are also presented accordingly. B) Sequential polar body (PB) 1 and PB2 analysis for maternal mutation IVS1-110, with five linked markers in 9 oocytes, predicting 4 mutant and 5 normal oocytes, 2 of them origination from heterozygous PB1 and mutant PB2, representing an good candidates for transfer. C) HLA typing by short tandem repeats (STRs) along with mutation analysis was performed on blastomeres from all nine embryos, two of which (\#4 and \#8) was predicted to be mutation free and also HLA match to the affected sibling 2.1. Another matched carrier of maternal mutation (embryo \#11) was frozen. Both normal matched embryos were transferred, resulting in a singleton pregnancy and birth of thalassemia free child matched to the affected siblings. The bone marrow of this child was transplanted to the thalassemic sibling (2.1), with a successful hematopoietic reconstitution and total cure of the thalassemic child. 
Table 1. Preimplantation genetic diagnosis for hemoglobinopathies with HLA typing.

\begin{tabular}{|c|c|c|c|c|c|c|c|}
\hline Disease & Gene & \# Patient & \# Cycle & \# Transfers & $\begin{array}{l}\text { \#Embryo } \\
\text { transferred }\end{array}$ & Pregnancy & Birth \\
\hline $\begin{array}{l}\text { Hemoglobin- } \alpha \text { locus } 1 ; \text { HBAl } \\
\text { Hemoglobin- } \beta \text { locus; HBB }\end{array}$ & $\begin{array}{l}H B A 1 \\
H B B\end{array}$ & 148 & 237 & 206 & 437 & 78 & 86 \\
\hline Sickle cell anemia & $H B B$ & 61 & 92 & 83 & 170 & 34 & 30 \\
\hline Subtotal & & 209 & 329 & 289 & 607 & 112 & 116 \\
\hline HLA + hemoglobin- $\beta$ locus; HBB & $H B B$ & 61 & 137 & 78 & 121 & 20 & 15 \\
\hline Sickle cell anemia + HLA & $H B B$ & 6 & 9 & 5 & 9 & 1 & 1 \\
\hline Subtotal & & 67 & 146 & 82 & 130 & 21 & 16 \\
\hline TOTAL & & 276 & 475 & 372 & 737 & 133 & 132 \\
\hline
\end{tabular}

fected embryos in 230 of them, yielding 72 unaffected HLA matched pregnancies and the birth of 62 HLA matched children, potential donors for their affected siblings.

The other successful experience of 148 PGD cases performed for thalassemia with HLA typing has recently been reported from Istanbul Center. ${ }^{13}$ Of 44 transplanted children, 40 showed a total hematopoietic reconstitution, while the graft failure occurred only in 4 of them. With the current progress in treatment of hemoglobinopathies, PGD may have an increasing impact on the decision of the well-treated patients with a carrier partner to reproduce. In fact, the life expectancy of the patients with hemoglobin disorders has been dramatically improved in last 10 year, with the success of radical treatment by stem cell transplantation, so PGD for hemoglobinopathies is already a practical option, available for a wider application in those communities where these genetic diseases are prevalent. It may be expected that a combined PGD and preimplantation HLA testing will in future be more widely used for further improvements in radical therapy of hemoglobinopathies by stem cell transplantation.

\section{Conclusions}

Despite ethical issues involved in preimplantation HLA typing, there is an increase of the attractiveness of this option for the couples with affected children requiring HLA compatible stem cell transplantation, providing a practical solution for those couples who would like to have another child anyway. This and other new indications make preimplantation testing for thalassemia a genuine alternative to conventional prenatal diagnosis, providing patients with important prospect not only to avoid an inherited risk without facing termination of pregnancy, but also to establish a pregnancy with particular genetic parameters, which may also benefit the affected member of the family.

\section{References}

1. Goossens V, Traeger-Synodinos J, Coonen E, et al. ESHRE PGD Consortium data collection XI: cycles from January to December 2008 with pregnancy follow-up to October 2009. Hum Reprod 2012;27:1887-911.

2. Kuliev A. Practical preimplantation genetic diagnosis. New York, London, Heidelberg: Springer; 2012.

3. Kuliev A, Rechitsky S, Verlinsky 0, et al. Preimplantation diagnosis of thalassemia. J Assist Reprod Genet 1998;15:219-25.

4. Kuliev A, Rechitsky S, Verlinsky 0, et al. Birth of healthy children after reimplantation diagnosis of thalassemias. J Assist Reprod Genet 1999;16:207-11.

5. Kanavakis E, Vrettou C, Palmer G, et al. Preimplantation genetic diagnosis in 10 couples at risk for transmitting beta-thalassemia major: clinical experience including initiation of six singleton pregnancies. Prenat Diagn 1999;19:1217-22.
6. Rechitsky S, Kuliev A, Tur-Kaspa I, et al. Preimplantation HLA typing with preimplantation genetic diagnosis. Reprod Biomed Online 2004;6:488-93.

7. Van deVelde H, Georgiou I, De Rycke M, et al. Novel universal approach for preimplantation genetic diagnosis of b-thalassemia in combination with HLA matching of embryos. Hum Reprod 2004;19:700-08.

8. Kahraman S, Karlilaya G, Sertyel S, et al. Clinical aspects of preimplantation genetic diagnosis of single gene disorders combined with HLA typing. Reprod Biomed Online 2004;9:529-32.

9. Kuliev A, Rechitsky S, Verlinsky 0 , et al. Preimplantation diagnosis and HLA typing for hemoglobin disorders. Reprod Biomed Online 2005;11:362-70.

10. Kuliev A, Pakhalchuk T, Verlinsky 0, Rechitsky S. Preimplantation genetic diagnosis for hemoglobinopathies. Hemoglobin 2011;35:547-55.

11. Kuliev A, Packalchuk T, Verlinsky 0, Rechitsky S. Preimplantation diagnosis: efficient tool for human leukocyte antigen matched bone marrow transplantation for thalassemia. Thal Rep 2011;1:e1.

12. Gaziev J, Lucarelli G. Allogeneic cellular gene therapy for hemoglobinopathies. Hemat Oncol Clin N Am 2010;24:1145-63.

13. Kahraman S. PGD for HLA: clinical outcome of HLA compatible transplantation following PGD. Reprod Biomed Online 2013;26: S9-S10. 